\title{
Effect of Bartonella spp. on the Freezing Tolerance in Female Ixodes scapularis
}

\author{
Emily Curry ${ }^{1}$, Amal El Nabbout ${ }^{1}$, James Kho ${ }^{1}$, Kyle D’Entremont ${ }^{1}$, Frederica Jacks ${ }^{1} \&$ Tatiana Rossolimo ${ }^{1}$ \\ ${ }^{1}$ Department of Biology, Dalhousie University, 1355 Oxford St., Halifax, Ns, B3h 4r2 Canada \\ Correspondence: Emily Curry, Department of Biology, Dalhousie University, 1355 Oxford St., Halifax, Ns, B3h \\ 4r2 Canada. E-mail: Em598226@Dal.Ca
}

Received: April 16, 2017

Accepted: April 26, 2017

Online Published: June 19, 2017

doi:10.5539/ijb.v9n3p65

URL: https://doi.org/10.5539/ijb.v9n3p65

\begin{abstract}
The aim of the current study was to determine the effect of Bartonella spp. on the freezing tolerance of engorged and non-engorged female Ixodes scapularis (Say). Bartonella is a genus of pathogenic vector-transmitted gram-negative bacteria, implicated in zoonotic disease transmission. I. scapularis ticks collected throughout Nova Scotia, Canada underwent freezing tolerance testing. DNA extraction, PCR and gel electrophoresis were then conducted to determine the presence and prevalence of Bartonella spp. in the experimental population. The experimental results demonstrated that the changes in the freezing tolerance as a result of the presence of Bartonella spp. were not statistically significant. The infection rate observed in the experimental population was $58.61 \%$; this was significantly higher comparable to the infection rate found in earlier research. It was concluded that Bartonella spp. had no altering physiological effect on Ixodes scapularis.
\end{abstract}

Keywords: Ixodes scapularis, Bartonella spp., Freezing Tolerance, Prevalence

\section{Introduction}

\subsection{Bartonella}

Bartonella is the sole genus of the family Bartonellaceae, and is a genus of pathogenic gram-negative bacteria. Bartonella spp. are facultative intracellular parasites that localize in the endothelial cells and the red blood cells (Chomel \& Boulouis, 2005). The genus is made up of 26 distinct species (Zaher et al., 2002). Of the 26 species 54\% are implicated in zoonotic diseases (Ricardo, 2012). Bartonella spp. bacteria are the underlying cause of a multitude of illness such as: Cat Scratch Fever, Trench Fever and Carrion Disease (Cotté et al., 2008). Bartonella $s p p$. are transmitted by vectors such as ticks, fleas, mosquitos and sandflies. There is currently no evidence that ticks can transmit Bartonella spp. pathogens to humans (Cotté et al., 2008).

\subsection{Ixodes Scapularis and Distribution}

The experimental organism: Ixodes scapularis, commonly referred to as the deer tick, is a species of hard tick belonging to the class Arachnida and family Ixodidae. These ticks are obligate hematophagous arthropods that are capable of transmitting pathogenic agents, such as virus, bacteria and protozoa, to their host through their hypostome (Shaw et al., 2001). Similar to other species of hard ticks I. scapularis has a two year life cycle, and overwintering at both the nymph and adult life stages (Burks et al., 1996). Additionally, at each life stage: larva, nymph, and adult, the tick will attach and feed on a singular distinctive host. (Parola \& Raoult, 2001). I. scapularis are distributed throughout Eastern North America. Furthermore, distribution and population size are linked to changes in climate. The length of each season as well as the daily variability in temperature and humidity play a key role in the survival, development, and activity of ticks (Lindgren \& Jaenson, 2006). As a result the density of the tick population in a given environment is the result of the combined effect of climatic and environmental conditions that have occurred over several years (Lindgren \& Jaenson, 2006).

Current research suggests that the presence of a bacterial agent, such as Bartonella spp., may alter the physiology and the behavior of Ixodes spp. (Herrmann \& Gern, 2010). Consequently the tests conducted in the current study were selected based on their ability to indicate physiological changes as a result of bacterial infection. The test conducted in the current study was inoculative freezing tolerance. The inoculative freezing tolerance testing was completed in accordance with previous studies on the aspects of cold hardiness in ixodid ticks (Burks et al., 1996). 
As the tick body reaches its super-cooling point an increase in temperature is produced by the crystallization of liquids within the body of the tick, which releases heat. The results of this test would provide information on a potential cause of overwintering mortality in Ixodes spp. (Burks et al., 1996). The methods were interpreted and adapted from research completed by Hermann et al. (2009).

The focus of the current study is on the effect of Bartonella spp. on the freezing tolerance in Ixodes scapularis. The current hypothesis states that the presence of Bartonella spp. in the tick will positively affect the freezing tolerance. The future implications of this research will help with monitoring the changes in movement and increasing abundance of ticks in and the potential role Bartonella infections have in the environmental expansion of $I$. scapularis.

\section{Methods}

\subsection{Freezing Tolerance}

The freezing tolerance testing of the ticks was completed using a VWR-1197P cooling bath. The ticks were attached to a thermocouple using petroleum jelly and placed into a cooling bath of Ethylene glycol solution composed of $60 \%$ ethylene glycol and $40 \%$ water which freezes at $-45^{\circ} \mathrm{C}\left(-49^{\circ} \mathrm{F}\right)$. The freezing process began at $4^{\circ} \mathrm{C}$, and the temperature continued to drop by $1{ }^{\circ} \mathrm{C}$ every one minute until the cooling bath reached $-30^{\circ} \mathrm{C}$. Data was collected using LoggerPro 16 computer software. LoggerPro 16 recorded a spike at the freezing point temperature of the tick. This process was repeated for each tick within the two categories: engorged females and non-engorged females.

\subsection{Cutting}

The ticks were cut into two halves down the sagittal plane. This process was completed on a surface sanitized with a $70 \%$ ethanol solution, with a sanitized scalpel. Each half was placed in its own properly labelled Eppendorf tube. One half was used for DNA extraction, the other was used for other research. The tick carcasses were stored in a freezer at $-20^{\circ} \mathrm{C}$ until DNA extraction was completed.

\subsection{DNA Extraction}

The DNA extraction required 50 $\mu 1$ of Aquagenomic (Multitarget Pharmaceuticals, Colorado, USA) solution to be added to the half tick sample in the Eppendorf tube. The sample was then homogenized with a micropestle for approximately 30 seconds to one minute. The homogenized sample was then incubated in a $60^{\circ} \mathrm{C}$ water bath for 45 minutes. During this time a second Eppendorf tube was labelled with the same ID, and was prepared with $50 \mu 1$ of Isopropanol. After the 45 minutes had elapsed the tube was vortex for 10 seconds. The tube was then placed in the centrifuge at $13 \mathrm{k} \mathrm{rpm}$ for four minutes. The tube was removed from the centrifuge and the supernatant of the sample was pipetted into the second Eppendorf tube containing Isopropanol. The new sample was then centrifuged for four minutes at $13 \mathrm{k} \mathrm{rpm}$. This process formed a pellet in the bottom of the solution. The supernatant was decanted and the pellet was rinsed with $50 \mu 1$ of $70 \%$ ethanol solution. The ethanol was then decanted, and any leftover ethanol was pipetted out of the Eppendorf tube. The sample was left to dry for 15 minutes. At this point $50 \mu 1$ of double distilled water $\left(d d \mathrm{H}_{2} \mathrm{O}\right)$ was added. The sample was incubated for one hour in a $60^{\circ} \mathrm{C}$ water bath. The samples were stored in a freezer at $-20^{\circ} \mathrm{C}$ until the PCR was conducted. This process was completed for each tick sample.

\subsection{Polymerase Chain Reaction}

A master mix was prepared using $8.5 \mu 1_{\mathrm{nf}} \mathrm{H}_{2} \mathrm{O}, 12.5 \mu 1$ of GoTaq Green (Promega, Maddison, USA), and $1.0 \mu 1$ of each of the following primer: P-12B for the sequence 5'-CGGGATCCCGAGATGGCTTTTGGAGATTA-3'and P-24E for the sequence 5'-GGAATTCCCTCCTTCAGTTAGGCTGG-3' (Relman et al., 1990), adapted from Eskow, Rao, and Mordechai 2001 for each sample. Control measures were also put into place when calculating the

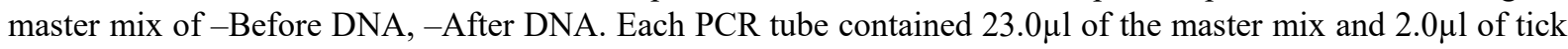
DNA, or water in the instance of the two controls. PCR for the detection of Bartonella spp. done based on the coded section 16S-rRNA. The PCR samples were prepared in a Biological Safety Cabinet, all materials excluding GoTaq Green, Primers and DNA are placed under UV for five minutes for sterilization. The expected amplicon of the primer was at $279 \mathrm{bp}$.

The setting of the The Engine Tetrad PTC-225 thermocycler (Biorad, Mountain View, USA) used to amplify the DNA with the $16 S r R N A$ primers $\mathrm{P} 24 \mathrm{E}$ and $\mathrm{P} 12 \mathrm{~B}$ was $94^{\circ} \mathrm{C}$ for three minutes, 40 cycles of $94{ }^{\circ} \mathrm{C}$ for 30 seconds, $56{ }^{\circ} \mathrm{C}$ for 45 seconds, and $72{ }^{\circ} \mathrm{C}$ for 45 seconds followed by a final elongation period at $72{ }^{\circ} \mathrm{C}$ for 10 minutes. 


\subsection{Gel Electrophoresis}

Gel electrophoresis was conducted on the PCR products under the following conditions: $2.0 \%$ agarose gel dyed using Gel Green Nucleid Acid Strain (Biotium, Hayward, USA), and run using a Tris-Borate EDTA (TBE) buffer for one hour at 116v. The DNA ladder (FroggaBio, Toronto, Canada) used was a 100bp ladder. The gels were visualized using a UV plate and photos were taken of the gel for analysis and reference. The collected data was then analyzed using comparison column graphs, as well as statistical T-tests.

\section{Results}

\subsection{Infection Rate}

The rate of infection of Bartonella spp. in Ixodes scapularis ticks, determined by the completion of PCR at $16 S-r R N A$ from the Bartonella genus was approximately $64 \%: 53.13 \%$ in a sample of 64 engorged females, and $64.10 \%$ in a sample of 39 non-engorged females.

\subsection{Inoculative Freezing}

In both experimental categories the infected and non-infected individuals froze at approximately the same temperature. In the engorged females the infected individuals $(-15.82 \pm 3.10)$ exhibited an almost identical inoculative freezing point to the uninfected individuals $(-15.95 \pm 3.98)(\mathrm{t}=0.88 .0 .50>\mathrm{p}>0.30$; Fig 1$)$. In the non-engorged females there was approximately a two degree difference in the inoculative freezing point between the infected and non-infected individuals. For the infected individuals $(-14.87 \pm 3.19)$ and for the non-infected individuals $(-16.85 \pm 2.95)(\mathrm{t}=0.06 .0 .80>\mathrm{p}>0.70 ;$ Fig 2$)$. The null hypothesis which states that there was no difference in the freezing tolerance when infected with Bartonella spp. was therefore failed to be rejected.

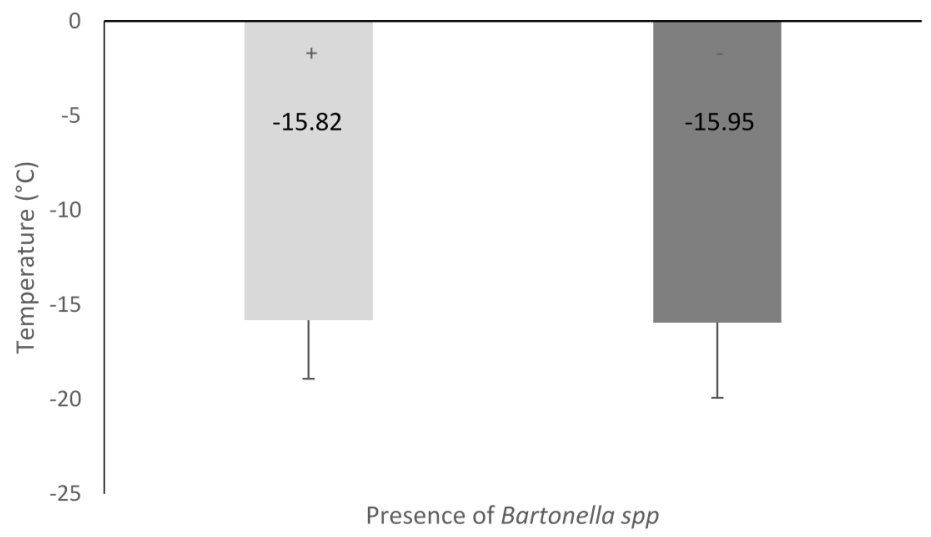

Figure 1. Comparison of the average (+ SD) freezing tolerance of the Ixodes scapularis sample engorged female that are positive and negative for Bartonella spp. pathogens. Data was collected throughout 2015-16 across Nova Scotia, Canada

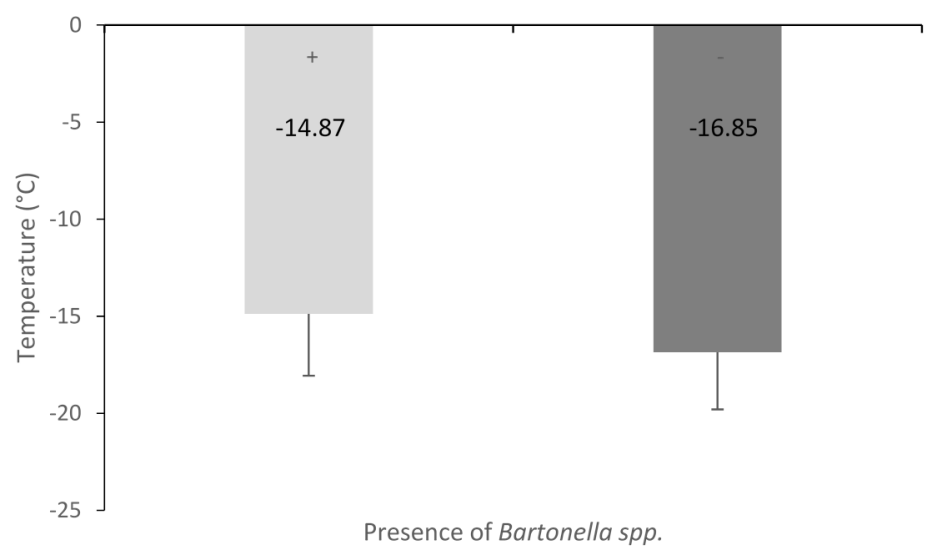

Figure 2. Comparison of the average (+ SD) freezing tolerance of the Ixodes scapularis sample non-engorged females that are positive and negative for Bartonella spp. pathogens. Data was collected throughout 2015-16 across Nova Scotia, Canada 


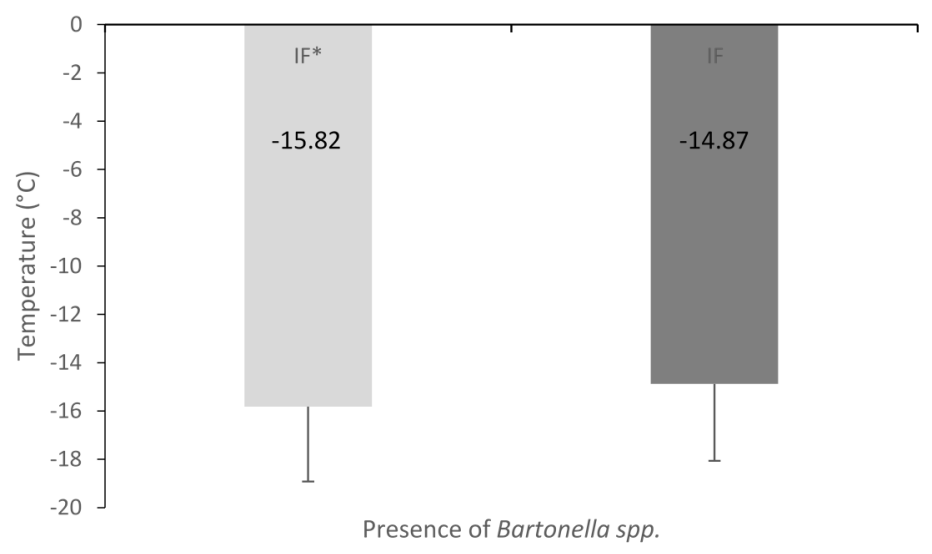

Figure 3. Comparison of the average (+ SD) freezing tolerance of the Ixodes scapularis samples female and engorged female that are positive for Bartonella spp. pathogens. Data was collected throughout 2015-16 across Nova Scotia, Canada

\section{Discussion}

The main findings of the current study demonstrated the presence of Bartonella spp. in I. scapularis did not result in a statically significant change in freezing tolerance (Figures 1 and 2). Consequently the null hypothesis which stated that there would be no change in freezing tolerance as a result of the presence of Bartonella spp. was failed to be rejected.

The experimental population was separated into two distinct categories based on feeding state: Engorged females and non-engorged females. Consequently the infection rate was calculated for the two experimental group, as well as the overall infection rate of the experimental population. The infection rate of the engorged females was $53.13 \%$, and non-engorged females $64.10 \%$. The overall infection rate of the total experimental population was 59 out of the 103 ticks with a results of $58.61 \%$. The infection rate achieved in the current research was considerably higher compared to previous research conducted by Adelson et al. (2004) on the presence of Bartonella spp. in I. scapularis in northern New Jersey. The results of this study indicated an infection rate of 37 ticks out of a sample size of 107 , or $34.5 \%$. Additionally, when compared to other previous research the results of the current study were significantly higher. Research completed in 2001 in California found that the infection rate of a similar species, Ixodes pacificus, was 19.2\% (Chang et al., 2001). Moreover, similar research completed in 2002 on Ixodes pacificus in California found a lower infection rate of $11.6 \%$ (Chang et al., 2002). Consequently, this leads to the review of potential sources of error in the experimental design and execution.

The potential sources of error that may have impacted the significance of the result are as follows: Firstly, the small sample size has the potential to skew the results of the infection rate. The sample size contained only 70 individuals, comparably to earlier research which contained a larger sample sizes of upwards of 900 individuals (Hermann, Voordouw, \& Gern, 2013). Moreover, P-12B and P-24E are non-specific primers. These primers amplify the section 16S-rRNA. The amplification of this portion of DNA can also be indicative of another genus of gram-negative bacteria: Mezorhizobium spp. (Dillon \& Iredell, 2005). Therefore, the positive results have the potential to be either Bartonella spp. or Mezorhizobium spp.; thereby, altering the infection rate achieved in the current study (Dillon \& Iredell, 2005).

Though not statistically significant the results of the current study provide anecdotal evidence that the presence of Bartonella spp., unlike Borrelia burgdorferi, does not affect freezing tolerance in I. scapularis. When reviewing the results of the freezing tolerance testing for the two experimental groups it was found that the individuals who tested positive for Bartonella spp. froze at approximately the same temperature. In the case of the engorged female group the temperature increase was of less than 0.15 of $1{ }^{\circ} \mathrm{C}$ (Table 1). Moreover, in the non-engorged females the temperature difference was less than $2^{\circ} \mathrm{C}$ (Table 2). This is a novel finding based on the ticks found in Nova Scotia, Canada.

Based on the results of the current study it was deduced that the presence of Bartonella spp. pathogens in I. scapularis did not produce any altered physiological effects in regards to freezing tolerance. Meaning, the presence of Bartonella spp. did not affect the freezing tolerance of I. scapularis. These results can be compared to earlier research conducted by Hermann et al. (2009) which found that the presence of Borrelia burgdorferi, the 
causative agent of Lyme disease, alters the physiology of Ixodes spp; thereby, affecting the freezing tolerance. The comparison of the two studies raises additional research questions, such as which bacteria strains produce physiologic effects, the effect of the physiological changes on the ticks behavior, as well as how changes in physiology effect distribution.

The current study documented no statistically significant difference in the freezing tolerance in Ixodes scapularis infected with Bartonella spp. disproving the initial hypothesis. I. scapularis are found throughout Nova Scotia; consequently, a greater understanding of the effects of Bartonella spp. on ticks is essential. Moreover, further research into other bacterial infections in ticks, as well as the rate of co-infection would provide a greater understanding of physiological changes as well as consequential alterations in distribution. Therefore, my final recommendation would be for additional sampling and testing to create a more accurate survey of the distribution of I. scapularis, as well as the bacterial infections found in the Nova Scotia tick population.

\section{Acknowledgements}

This research was supported by Dalhousie University. We thank Dr. P. Bentzen for access to research space and Dr. S. Adamo and laboratory colleagues for discussions and help.

\section{References}

Adelson, M. E., Rao, R. V. S., Tilton, R. C., Cabets, K., Eskow, E., Fein, L., ... \& Mordechai, E. (2004). Prevalence of Borrelia burgdorferi, Bartonella spp., Babesia microti, and Anaplasma phagocytophila in Ixodes scapularis ticks collected in Northern New Jersey. Journal of Clinical Microbiology, 42(6), 2799-2801. https://doi.org/10.1128/JCM.42.6.2799-2801.2004

Burks, C. S., STEWART, R. L., Needham, G. R., \& LEE, R. E. (1996). The role of direct chilling injury and inoculative freezing in cold tolerance of Amblyomma americanum, Dermacentor variabilis and Ixodes scapularis. Physiological Entomology, 21(1), 44-50.

Chang, C. C., Chomel, B. B., Kasten, R. W., Romano, V., \& Tietze, N. (2001). Molecular evidence of Bartonella spp. in questing adult Ixodes pacificus ticks in California. Journal of Clinical Microbiology, 39(4), 1221-1226. https://doi.org/10.1128/JCM.39.4.1221-1226.2001

Chang, C. C., Hayashidani, H., Pusterla, N., Kasten, R. W., Madigan, J. E., \& Chomel, B. B. (2002). Investigation of Bartonella infection in ixodid ticks from California. Comparative immunology, microbiology and infectious diseases, 25(4), 229-236. https://doi.org/10.1016/S0147-9571(02)00012-7

Chomel, B. B., \& Boulouis, H. J. (2005). Zoonoses dues aux bactéries du genre Bartonella: nouveaux réservoirs? nouveaux vecteurs? Bulletin de l'Académie Nationale de Médecine, 189(3), 465-480.

Cotté, V., Bonnet, S., Le Rhun, D., Le Naour, E., Chauvin, A., Boulouis, H. J., ... \& Vayssier-Taussat, M. (2008). Transmission of Bartonella henselae by Ixodes ricinus. Emerging infectious diseases, 14(7), 1074.

Dillon, B., \& Iredell, J. (2005). Potential limitations of the 16S-23S rRNA intergenic region for molecular detection of Bartonella species. Journal of Clinical Microbiology, 43(9), 4921-4922. https://doi.org/10.1128/ JCM.43.9.4921-4922.2005

Eskow, E., Rao, R. V. S., \& Mordechai, E. (2001). Concurrent infection of the central nervous system by Borrelia burgdorferi and Bartonella henselae. Archive Neurology, 58, 1357-1363.

Herrmann, C., \& Gern, L. (2010). Survival of Ixodes ricinus (Acari: Ixodidae) under challenging conditions of temperature and humidity is influenced by Borrelia burgdorferi sensu lato infection. Journal of Medical Entomology, 47(6), 1196-1204. https://doi.org/10.1603/ME10111

Herrmann, C., Voordouw, M. J., Gern, L. (2013). Ixodes ricinus ticks infected with the causative agent of lyme disease, Borrelia burgdorferi sensu lato, have higher energy reserves. International Journal of Parasitology, 43(6), 477-83. https://doi.org/10.1016/j.jpara.2012.12.010

Lindgren, E., \& Jaenson, T. G. T. (2006). Lyme borreliosis in Europe: influences of climate and climate change, epidemiology, ecology and adaption measures. World Health Organization, 1, 4-25.

Maggi, R. G., Mozayeni, B. R., Pultorak, E. L., Hegarty, B. C., Bradley, J. M., Correa, M., \& Breitschwerdt, E. B. (2012). Bartonella spp. bacteremia and rheumatic symptoms in patients from Lyme disease-endemic region. Emerging infectious diseases, 18(5), 783-791. https://doi.org/10.3201/eid1805.111366

Parola, P., \& Raoult, D. (2001). Ticks and tickborne bacterial diseases in humans: an emerging infectious threat. Ticks and Tick Borne Diseases, 32, 897-928. https://doi.org/10.1086/319347 
Relman, D. A., Loutit, J. S., Schmidt, T. M., Falkow, S., \& Tompkins, L. S. (1990). The agent of bacillary angiomatosis: an approach to the identification of uncultured pathogens. New England Journal of Medicine, 323(23), 1573-1580. https://doi.org/10.1056/NEJM199012063232301

Shaw, S. E., Day, M. J., Birtles, R. J., \& Breitschwerdt, E. B. (2001). Tick-borne infectious diseases of dogs. TRENDS in Parasitology, 17(2), 74-80. https://doi.org/10.1016/S1471-4922(00)01856-0

Zaher, Z., Zhongxing, L., \& Didier, R. (2002). Genetic classification and differentiation of Bartonella species based on comparison of partial ftsZ gene sequences. Journal of Clinical Microbiology, 40(10), 3614-3647. https://doi.org/10.1128/JCM.40.10.3641-3647.2002

\section{Copyrights}

Copyright for this article is retained by the author(s), with first publication rights granted to the journal.

This is an open-access article distributed under the terms and conditions of the Creative Commons Attribution license (http://creativecommons.org/licenses/by/4.0/). 